Check for updates

Cite this: RSC Adv., 2019, 9, 10345

\title{
Influence of surface tension-driven network parameters on backflow strength $\dagger$
}

\author{
Yonghun Lee, Islam Seder and Sung-Jin Kim (D)*
}

Surface tension-driven flow is widely used, owing to its spontaneous motion, in microfluidic devices with single channel structures. However, when multiple channels are used, unwanted backflow often occurs. This prevents precise and sophisticated solution flow, but has been rarely characterized. We hypothesize that, with an analytical model, the parameters that influence backflow can be systematically characterized to minimize the backflow. In a microfluidic network, inlet menisci and channels are modeled as variable pressure sources and fluidic conductors, respectively. Through the model and experiment, the influence of each network element on the backflow strength is studied. Backflow strength is affected by the interplay of multiple inlet-channel elements. With the decrease (increase) of the fluidic channel conductance (inlet size), the backflow pressure of the corresponding inlet decreases. On the other hand, backflow volume reaches its peak value during the radius change of the corresponding inlet. In networks consisting of five inlet-channel elements, backflow pressure decreases with increasing step number. Our results provide the foundations for microfluidic networks driven by the Laplace pressure of inlet menisci.

Received 27th November 2018 Accepted 26th March 2019

DOI: $10.1039 / \mathrm{c} 8 \mathrm{ra09756a}$

rsc.li/rsc-advances generate inlet pressures (i.e., the Laplace pressure). Importantly, this pressure significantly affects the motion of the flow. When multiple channels are prefilled with solutions and a solution is injected to an inlet, the inlet of the solution has high Laplace pressure and pushes other solutions to their corresponding inlets. The ideal case is that the initial flow direction in each channel is maintained until the flow stops. However, the flow direction in several channels can often reverse unexpectedly, resulting in backflows. Even when the filling of channels occurs by capillary action, backflow occurs. For example, when liquid fronts of two solutions merge at Yjunction and move to the junction downstream, one solution from an inlet unexpectedly moves back to the other inlet through the Y-junction. ${ }^{19-22}$ Such unwanted motion occurs by the imbalance of the Laplace pressure of inlets. To date, such unwanted backflows have been reported in the process of immunoassay, ${ }^{18,19}$ micromixing, ${ }^{20}$ blood typing, ${ }^{21}$ and cell-based assay. $^{22}$ In the case of unexpected backflows in a surface tension-driven bioassay chip, the designed sequence of fluidic motion is changed unintentionally, and the detection of targets will fail. Thus, reducing backflow in network channels is a crucial challenge that must be overcome for such applications. Our recent study showed that backflow results from the difference between the time constant ratios of a network's inletchannel elements. ${ }^{23}$ However, the contributions of network elements including channel fluidic conductance, inlet radius, and pressure to backflow strength were not analyzed in detail.

In this paper, we study the effect of each element of a surface tension-driven network on backflow strength. We model inlet 
menisci as variable pressure sources, and channels as fluidic conductors. Using the model, we characterize how the fluidic conductance of channels, and the radius and initial pressures of inlets, influence backflow strength. This analysis systematically explains the complex relations between network elements that affect backflow. Our model is first applied to characterize backflow in a surface tension-driven network with three inlets, and then extended to a network with five inlets.

\section{Working process and theoretical modeling}

In this section, we explain the process of capillary filling and backflow generation, and then discuss the corresponding theoretical model. Backflow can occur in a channel network consisting of multiple channels, Y-junctions, and multiple branched junctions. During the merging process of the solutions at the junctions, backflows occur by the pressure difference of inlets. To describe the basic process of backflow generation, we used a network with three inlets and three channels. This is the minimum element condition that can describe the backflow in a channel network. Three steps are used to fill the network and generate the backflow. Fig. 1a depicts the fluidic motion in the network in the last step; the motion is driven by the Laplace pressure of the inlet menisci. Fig. 1b presents each step. In step 1, solution 1 (green) injected at inlet 1 stops at a capillary valve ${ }^{24}$ of channel 1 . In step 2, solution 2 (red) injected at inlet 2 moves through channel 2 to channel 1. The fluidic motion continues until the two inlet pressures equilibrate. Backflow does not occur because only two inlet pressures are involved for fluidic motion. Step 3 consists of two parts (steps $3 \mathrm{a}$ and $3 \mathrm{~b}$ ). In step $3 \mathrm{a}$, solution 3 (clear) injected at inlet 3 moves into channels 1 and 2 . Then in step $3 b$, the flow direction in either channel 1 or 2 is reversed and backflow may occur. The right panels of Fig. 1b present two cases with different backflow strengths. In case 1 , solution 2 exits inlet 2 and moves back from channel 2 to channel 1, resulting in the strong backflow of solution 2 . If channel 1 is the target channel that each solution needs to move into sequentially, then the sequence is disrupted in step 3 by the backflow. In case 2 , although the backflow of solution 2 occurs, the flow stops in channel 2 without entering channel 1, and the backflow strength is weak. Thus, the three solutions were successfully injected into channel 1 in a sequential manner.
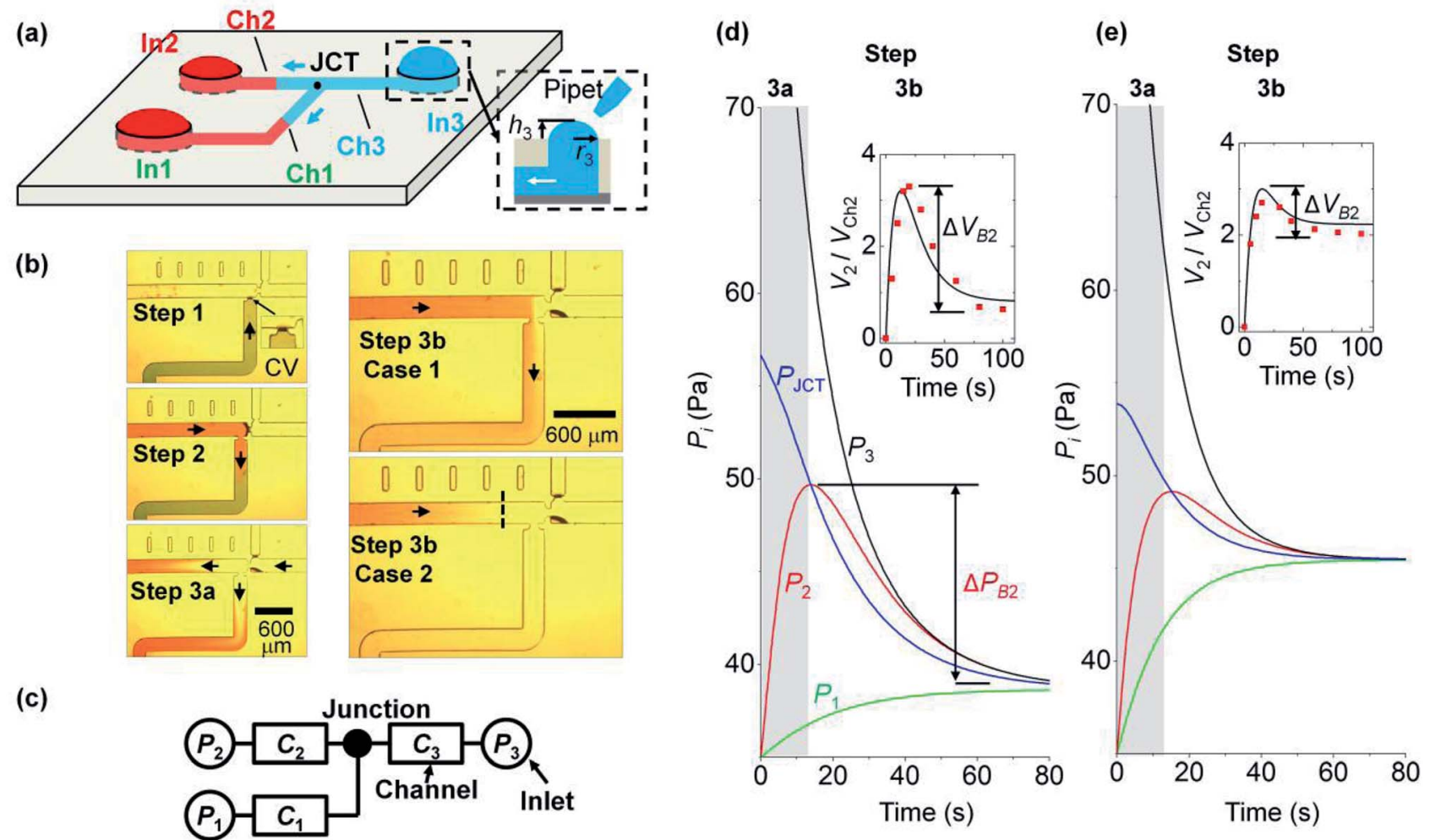

Fig. 1 Surface tension-driven network showing backflow. (a) Generation of fluidic motion by the pressure difference of inlet menisci. Inlets (In $i, i$ $=1$ to 3 ) are connected to each other through channels ( $\mathrm{Ch} i$ ) and the junction (JCT). The inset shows the cross-section of inlet 3 with inlet radius $r_{3}$ and meniscus height $h_{3}$. (b) Photographs showing backflow generation process. The process consists of three steps, and backflow occurs in step 3b. Cases 1 and 2 show strong and weak backflows, respectively. In step 3 , the initial pressures of inlet $i\left(P_{\mid i}\right)$ were the same for both cases with $P_{\mathrm{l} i}=35,35$, and $100 \mathrm{~Pa}$ ( $i=1$ to 3). (c) Circuit diagram of the capillary network. $P_{i}$ is the pressure of inlet $i$ with radius $r_{i}$, and $C_{i}$ is fluidic conductance of channel $i$. ( $d$ and e) Temporal change in inlet pressures in step 3. Lines and points are the theoretical and experimental values, respectively. Inlet $i$ has pressure $P_{i}$ in step 3. $\Delta P_{\mathrm{B} 2}$ is the backflow pressure of inlet 2 , and $\Delta V_{\mathrm{B} 2}$ is the normalized backflow volume. In (d), $r_{i}=2,1$, and $1 \mathrm{~mm}(i=1$ to 3$)$, and $C_{i}=9,9$, and $9\left(\times 10^{-12}\right) \mathrm{m}^{5} \mathrm{~N}^{-1} \mathrm{~s}^{-1} . \ln (\mathrm{e}), r_{i}=1.5,1$, and $1 \mathrm{~mm}$, and $C_{i}=12,9$, and $9\left(\times 10^{-12}\right) \mathrm{m}^{5} \mathrm{~N}^{-1} \mathrm{~s}^{-1}$. 
We modeled the process of backflow generation in a surface tension-driven network (Fig. 1c). The inlet menisci were modeled as variable pressure sources because the volume of each meniscus changes in each step, which temporally varies inlet pressures. The channels were modeled as fluidic conductors. The detailed derivation process is explained in Sections 1 and 2 of ESI. $\uparrow$ The pressure $\left(P_{i}\right)$ of inlet $i$ is obtained by

$$
P_{i}=\frac{4 \sigma h_{i}}{h_{i}^{2}+r_{i}^{2}}
$$

where $\sigma$ is the surface tension of a solution injected into inlet $i$, $h_{i}$ is the height of the convex meniscus of the solution at inlet $i$, and $r_{i}$ is the radius of inlet $i$ (see inset of Fig. 1a). For eqn (1), the shape of inlet meniscus is assumed to be a spherical cap. This is because gravitational forces on the inlet meniscus are negligible with the small Bond number, Bo $=\rho g L^{2} / \sigma<0.02$. Here, $L$ is the characteristic length and we used the meniscus height. Also, each inlet meniscus is assumed to be pinned to the rim of the inlet. We experimentally confirmed the meniscus pinning at the inlet rim under the condition of height-to-radius ratio $h_{i} / r_{\mathrm{i}}<0.5$ and solution contact angle $>49^{\circ}$ (Section 3 of ESI $\dagger$ ). We note that the condition $h_{i} / r_{i}<0.5$ is satisfied in normal surface tension-driven devices. Also, the condition of solution contact angle $>49^{\circ}$ is normally met, because the inlets of surface tension-driven devices typically use hydrophobic or moderately hydrophilic surfaces to confine the solution menisci to the rims of their inlets. Thus, our condition for meniscus pinning at the inlet rim can be considered as valid in most cases.

The pressure $\left(P_{\mathrm{JCT}}\right)$ at the junction of the channels is obtained by the analogy of Kirchhoff's current law and is given by

$$
P_{\mathrm{JCT}}=\frac{\sum_{i=1}^{j} C_{i} P_{i}}{\sum_{i=1}^{j} C_{i}}
$$

where $C_{i}$ is the fluidic conductance of channel $i$, which is the inverse of channel fluidic resistance. The change rate of $h_{i}$ is given by

$$
\frac{\mathrm{d} h_{i}(t)}{\mathrm{d} t}=\frac{2 C_{i}\left(P_{\mathrm{JCT}}-P_{i}\right)}{\pi\left(r_{i}^{2}+\left(h_{i}(t)\right)^{2}\right)}
$$

Eqn (3) is obtained by the condition where change rate of inlet volume is the same with the flow rate in the channel that connects the corresponding inlet and the junction. In addition, for eqn (2) and (3) we use Poiseuille's law, which neglects the contribution of flow inertia in the relation between pressure and flow rate. This is because the Reynolds number is small (Re $<0.5$ ) in our system. In eqn (3), the number of $h_{i}$ increases with increasing step number. For example, in step $2, h_{1}$ and $h_{2}$ are used; and in step $3, h_{1}, h_{2}$, and $h_{3}$ are used. The corresponding numbers of $P_{i}, r_{i}$, and $C_{i}$ also increase with increasing step number. To solve the simultaneous differential form of eqn (3), we measured the initial values of $h_{i}$ in each step, and used them as the initial condition of each step. The equations were numerically solved with MATLAB.

\section{Experimental}

We fabricated devices using soft lithography technique. ${ }^{25}$ The devices have two layers. The top layer was produced from hydrophobic polydimethylsiloxane (PDMS) with channel features, while the bottom layer was comprised of hydrophilic slide glass. The hydrophilicity of the slide glass was increased by an air plasma machine (Femto Science) so that the solutions injected at the inlets can spontaneously fill the channels and meet at the junction. The dimensions of inlets and channels were measured to calculate the fluidic conductance of each channel. The channel dimensions were in the range 60-100 $\mu \mathrm{m}$ $(h) \times 180-400 \mu \mathrm{m}(w) \times 1.4-50 \mathrm{~mm}(l)$, with inlet radii ranging from 1 to $2.5 \mathrm{~mm}$. The channel dimensions varied $C_{i}$ in the range of $7 \times 10^{-14}$ to $9 \times 10^{-12} \mathrm{~m}^{5} \mathrm{~N}^{-1} \mathrm{~s}^{-1}$. We used a stereo microscope (Olympus) and digital microscope (Dino-lite) to measure the meniscus height of each inlet. We used de-ionized water for the working solution and food dyes were added for flow visualization.

\section{Results and discussion}

\subsection{Backflow formation process}

We analyze backflow formation with our model. Fig. 1d and e show the theoretical change in pressures that correspond to cases 1 (strong backflow) and 2 (weak backflow) in Fig. 1b, respectively. The solutions move from high to low pressure positions. In step 3a (gray region in Fig. $1 \mathrm{~d}$ and e), the pressure condition is $P_{3}>P_{\mathrm{JCT}}>P_{2}\left(P_{\mathrm{I} 2}=P_{\mathrm{I} 1}\right)$. Thus, solution 3 at inlet 3 moves through the junction to inlets 1 and 2. Owing to the fluidic motions, $P_{3}$ decreased and $P_{1}$ and $P_{2}$ increased. In step $3 \mathrm{~b}$ (white region), $P_{2}$ becomes greater than $P_{\mathrm{JCT}}$, which reverses the flow direction of solution 2 from inlet 2 to the junction. Thus, because $P_{2}>P_{\mathrm{JCT}}>P_{1}$, solution 2 moves through the junction toward channel 1 and inlet 1 . We note that the backflow volume of solution 2 is different for the two cases. The insets of Fig. 1d and e present the volume ratio $\left(V_{2} / V_{\mathrm{Ch} 2}\right)$ of the inlet meniscus volume $\left(V_{2}\right)$ and channel 2 volume $\left(V_{\mathrm{Ch} 2}\right) \cdot V_{\mathrm{Ch} 2}$ was fixed at $30 \mathrm{~nL}$, and the initial value of $V_{2}$ was expressed as 0 by subtracting its initial volume from $V_{2}$. During backflow, $V_{2} /$ $V_{\mathrm{Ch} 2}$ decreases because solution 2 is released from inlet 2 . To quantify the backflow volume at inlet 2 , we define normalized backflow volume $\left(\Delta V_{\mathrm{B} 2}\right.$, see the insets) at inlet 2 as $\Delta V_{\mathrm{B} 2}=\left(V_{2 \mathrm{P}}-\right.$ $\left.V_{2 \mathrm{E}}\right) / V_{\mathrm{Ch} 2}$, where $V_{2 \mathrm{P}}$ and $V_{2 \mathrm{E}}$ are the volumes of inlet 2 meniscus at the peak and equilibrium states of $P_{2}$, respectively. The corresponding backflow pressure at inlet $2\left(\Delta P_{\mathrm{B} 2}\right)$ is defined in Fig. 1d. If $\Delta V_{\mathrm{B} 2}>1$ (i.e., $V_{2 \mathrm{P}}-V_{2 \mathrm{E}}>V_{\mathrm{Ch} 2}$ ), then the volume that goes out of inlet 2 is greater than the channel 2 volume, so the backflow of solution 2 moves through channel 2 to another channel. This case is shown in the inset of Fig. $1 \mathrm{~d}$, where $\Delta V_{\mathrm{B} 2}=$ $2.8>1$ (case 1 in Fig. 1b). In contrast, in the inset of Fig. $1 \mathrm{e}, \Delta V_{\mathrm{B} 2}$ $=0.6<1$, so solution 2 does not go out of channel 2 even for its backflow (case 2 in Fig. 1b). We analyzed how $P_{\mathrm{I} i}, r_{i}$, and $C_{i}$ influence the backflow strength at inlet 2 .

We note that the backflow shown in this system does not result from inertia of fluids. This is because the Reynolds number (Re), which is the ratio of inertia to viscous force, was 
$\mathrm{Re}<0.5$ in our system. For the inertia effect to be dominant, Re needs to be high. Indeed, the studies that reported backflows even in single channel systems had $\mathrm{Re}>10$, owing to large channel size and high inlet pressure driven by repeated injection of small drops. ${ }^{26-29}$ In addition, we used a solution containing food dye with the same concentration (3.6\% by weight). Thus, there was no flow induced by concentration gradient.

\subsection{Effect of fluidic conductance and channel size on the backflow}

We study the effect of the fluidic conductance of channel $i\left(C_{i}, i\right.$ $=1$ to 3 ) on the backflow. When one fluidic conductance was changed, the others were fixed at $7 \times 10^{-12} \mathrm{~m}^{5} \mathrm{~N}^{-1} \mathrm{~s}^{-1}$. In step 3 , inlet 3 releases solution 3 to the other inlets, inlet 2 is the place where backflow occurs, and inlet 1 takes the backflow solution. We analyze the backflow of inlet 2 under the change of $C_{i}$. Fig. 2a shows that high $C_{1}$ decreases $\Delta P_{\mathrm{B} 2}$ and $\Delta V_{\mathrm{B} 2}$. This behavior is explained by the fast rise of $P_{1}$ and its resultant small difference between $P_{1}$ and $P_{2}$. When $C_{1}$ increases, solution 3 injected from inlet 3 can easily move to inlet 1 rather than inlet 2. This is because comparatively high fluidic conductance of channel 1 allows rapid fluidic transport through channel 1 to inlet 1 . As a result, $P_{1}$ rapidly increases, according to the comparison $P_{1}$ in the gray region of Fig. $1 \mathrm{e}$ and $\mathrm{d}$. When $P_{1}$ becomes high, the difference between $P_{2}$ and $P_{1}$ decreases (step $3 \mathrm{~b}$ in Fig. 1d and e). Thus, the backflow that moves from inlet 2 toward inlet 1 decreases. Compared to $C_{1}$, high $C_{2}$ increases $\Delta P_{\mathrm{B} 2}$ and $\Delta V_{\mathrm{B} 2}$ (Fig. 2b). This is because, with high $C_{2}$, solution 3 can easily move through channel 2 to inlet 2 , thereby causing a more rapid increase in $P_{2}$ in step 3a. Similar to $C_{2}, C_{3}$ increases $\Delta P_{\mathrm{B} 2}$ and $\Delta V_{\mathrm{B} 2}$ (Fig. 2c). With high $C_{3}$, inlet 3 releases solution 3 more rapidly to the other channels through channel 3. This significantly increases $P_{2}$ in step 3 a and leads to high $\Delta P_{\mathrm{B} 2}$ and $\Delta V_{\mathrm{B} 2}$ in step $3 \mathrm{~b}$. In addition, when the size of channel 2 increases under constant condition of $C_{2}, \Delta V_{\mathrm{B} 2}$ decreases. This result is analyzed in Section 4 of ESI. $\dagger$

The backflow of inlet 2 by the change of $C_{i}$ can be collectively explained. High $C_{2}$ and $C_{3}$ increase backflow of inlet 2 . This is because, at step $3 \mathrm{a}$, solution 3 moves more rapidly to inlet 2 through channels 2 and 3 owing to high $C_{2}$ and $C_{3}$. On the other hand, high $C_{1}$ decreases the backflow of inlet 2 . This is because solution 3 moves more to inlet 1 rather than inlet 2 by the high fluidic conductance of channel 1 in step $3 \mathrm{a}$ and finally reduces the backflow of inlet 2 by the small pressure difference between inlets 1 and 2.

\subsection{Influence of inlet radius and inlet pressure on the backflow}

Here, we analyze the effect of inlet radius on the backflow of inlet 2. Fig. 3a shows how the three inlets influence $\Delta P_{\mathrm{B} 2}$. First, the left panel of Fig. 3a shows that high $r_{1}$ increases $\Delta P_{\mathrm{B} 2}$. This is because high $r_{1}$ makes the change of $P_{1}$ gradual (eqn (1)), leading to larger difference between $P_{1}$ and $P_{2}$ at the end of step 3a (Fig. 1d). As a result, $\Delta P_{\mathrm{B} 2}$ increases in step $3 \mathrm{~b}$. For the two cases of $C_{i}\left(7,7\right.$, and 7 ; and 5,9 , and $\left.10\left(\times 10^{-12}\right) \mathrm{m}^{5} \mathrm{~N}^{-1} \mathrm{~s}^{-1}\right)$, this trend is maintained. Second, the middle panel of Fig. 3a shows that high $r_{2}$ decreases $\Delta P_{\mathrm{B} 2}$. This is because increasing $r_{2}$ reduces the change rate of $P_{2}$ (eqn (1)), and $P_{2}$ gradually increases in step 3a (Fig. 1d). Thus, difference between $P_{1}$ and $P_{2}$
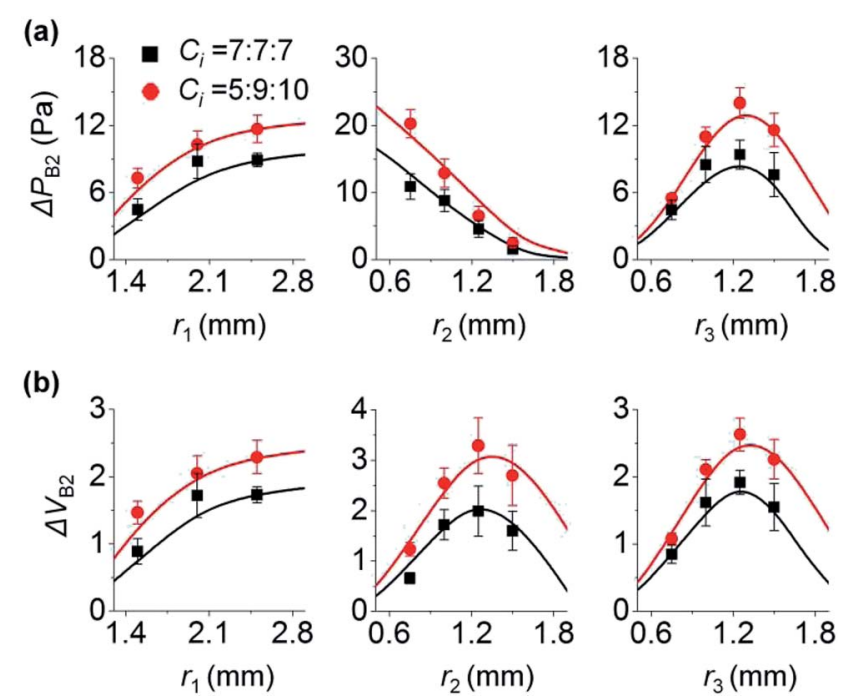

Fig. 3 Influence of inlet radius $r_{i}$ on the backflow of inlet 2. Unless otherwise noted, $r_{1}=2 \mathrm{~mm}$, and $r_{2}=r_{3}=1 \mathrm{~mm}$. In step 3, $P_{\mathrm{l} i}=35,35$, and $75 \mathrm{~Pa}$ ( $i=1$ to 3 ). $\Delta P_{\mathrm{B} 2}$ and $\Delta V_{\mathrm{B} 2}$ are shown in (a) and (b), respectively. Lines and points are the theoretical and experimental $(n=$ 3) values, respectively. (a)

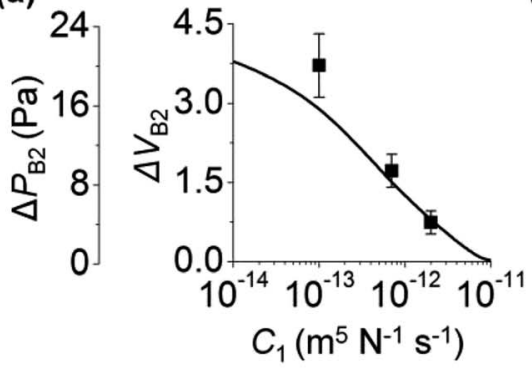

(b)

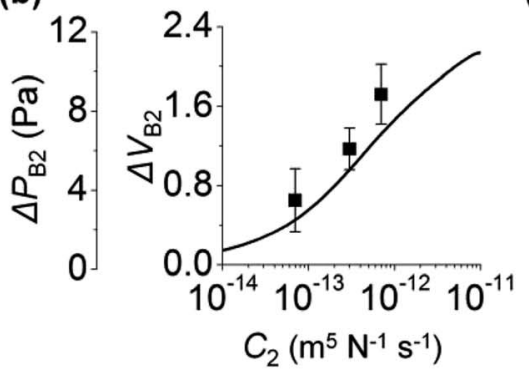

(c)

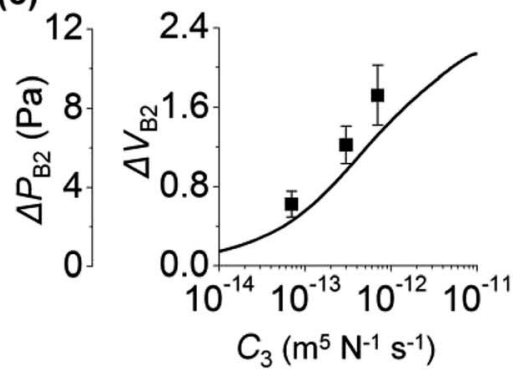

Fig. 2 Influence of $C_{i}$ on the backflow of inlet 2. When one fluidic conductance was changed, the others were fixed at $7 \times 10^{-12} \mathrm{~m}^{5} \mathrm{~N}^{-1} \mathrm{~s}^{-1}$. Here, $r_{i}=2,1$, and $1 \mathrm{~mm}$ ( $i=1$ to 3 ). In step $3, P_{1 i}=35,35$, and $75 \mathrm{~Pa}$. Variations of $\Delta P_{\mathrm{B} 2}$ and $\Delta V_{\mathrm{B} 2}$ by $C_{1}$ are shown in (a), by $C_{2}$ in (b), and by $C_{3}$ in (c). Lines and points are the theoretical and experimental $(n=3)$ values, respectively. 
at the end of step 3a in Fig. 1d becomes small, thereby reducing $\Delta P_{\mathrm{B} 2}$. Third, unlike the cases of $r_{1}$ and $r_{2}$, increasing $r_{3}$ does not monotonously vary $\Delta P_{\mathrm{B} 2}$ (right panel of Fig. 3a). When $r_{3}$ increases from 0.6 to $1.2 \mathrm{~mm}, \Delta P_{\mathrm{B} 2}$ increases. Herein, the initial pressure of inlet 3 was kept at $P_{\mathrm{I} 3}=75 \mathrm{~Pa}$ regardless of $r_{3}$. To meet the constant $P_{\mathrm{I} 3}$ condition even for increasing $r_{3}$, the initial meniscus volume of inlet 3 increases (Section 5 of ESI $\dagger$ ). Consequently, a larger amount of solution 3 goes from inlet 3 to inlet 2, thereby increasing $\Delta P_{\mathrm{B} 2}$. On the other hand, when $r_{3}$ becomes greater than $1.2 \mathrm{~mm}, \Delta P_{\mathrm{B} 2}$ decreases. We explain this result by using the difference between $P_{2}$ and $P_{\mathrm{JCT}}$ that influences the flow from inlet 2 to the junction. Backflow occurs in step $3 \mathrm{~b}$ only when $P_{2}>P_{\mathrm{JCT}}$ (Fig. 1d). Importantly, backflow decreases if $P_{2}-P_{\mathrm{JCT}}$ is small in step $3 \mathrm{~b}$; compare Fig. $1 \mathrm{~d}$ and e. High $r_{3}$ let $P_{\text {JCT }}$ stay at a high value through $P_{3}$ in step $3 \mathrm{~b}$ (Fig. 1d). This is because $P_{\text {JCT }}$ is considered as the weighted average of inlet pressures including $P_{3}$ (eqn (2)) and slow decrease of $P_{3}$ by high $r_{3}$ let $P_{\mathrm{JCT}}$ remain at a high value. Accordingly, pressure difference between $P_{\mathrm{JCT}}$ and $P_{2}$ decreases in step $3 \mathrm{~b}$, decreasing $\Delta P_{\mathrm{B} 2}$.

Collectively, when inlet 1 receives backflow from inlet 2, high $r_{1}$ decreases the difference between $P_{1}$ and $P_{2}$ at step 3a, leading to lower $\Delta P_{\mathrm{B} 2}$ at step $3 \mathrm{~b}$. Oppositely, high $r_{2}$ increases the difference between $P_{1}$ and $P_{2}$, thus increasing $\Delta P_{\mathrm{B} 2}$ at step $3 \mathrm{~b}$. Increasing $r_{3}$ rises $\Delta P_{\mathrm{B} 2}$ at first because of large volume of inlet 3 meniscus, and then reduces decreases $\Delta P_{\mathrm{B} 2}$ because of the low difference between $P_{2}$ and $P_{\mathrm{JCT}}$ at step $3 \mathrm{~b}$.

The change in $\Delta V_{\mathrm{B} 2}$ follows the trend of $\Delta P_{\mathrm{B} 2}$ when $r_{1}$ and $r_{3}$ vary (left and right panels of Fig. 3). As shown in Fig. 2, with $C_{i}$ variation, $\Delta V_{\mathrm{B} 2}$ and $\Delta P_{\mathrm{B} 2}$ changed in the same manner. On the other hand, $\Delta V_{\mathrm{B} 2}$ varied differently with $\Delta P_{\mathrm{B} 2}$ with the change in $r_{2}$ (middle panels of Fig. 3). $\Delta V_{\mathrm{B} 2}$ increases when $r_{2}$ increases from 0.6 to $1.2 \mathrm{~mm}$, and then decreases when $r_{2}>1.2 \mathrm{~mm}$. The increasing $r_{2}$ lets inlet 2 take more fluids from inlet 3 in step $3 \mathrm{a}$ (Section 6 of ESI $\dagger$ ). This allows inlet 2 to release a greater amount of backflow volume in step $3 \mathrm{~b}$ for $0.6<r_{2}<1.2 \mathrm{~mm}$. However, with increasing $r_{2}$, the reduction of $P_{2}$ becomes more gradual in step $3 \mathrm{~b}$ while $P_{\mathrm{JCT}}$ insignificantly changes by $r_{2}$. This leads to the reduced difference between $P_{\mathrm{JCT}}$ and $P_{2}$, thus decreasing $\Delta V_{\mathrm{B} 2}$ for $r_{2}>1.2 \mathrm{~mm}$ in step $3 \mathrm{~b}$.

To study the effect of the initial inlet pressures $\left(P_{\mathrm{I} i}\right)$ in step 3 on the backflow, $P_{\mathrm{I} i}$ values were varied and the backflow at inlet 2 was measured. Here, $P_{\mathrm{I} 3}$ was $75 \mathrm{~Pa}$ for different values of $P_{\mathrm{I} 2}$, with $P_{\mathrm{I} 2}=P_{\mathrm{I} 1}$. We used three sets of $C_{i}$, as shown in Fig. 4 . Under this condition, $\Delta P_{\mathrm{B} 2}$ and $\Delta V_{\mathrm{B} 2}$ unanimously increased with the increasing $P_{\mathrm{I} 3}-P_{\mathrm{I} 2}$. This is because at higher $P_{\mathrm{I} 3}-P_{\mathrm{I} 2}$, a comparatively larger amount of inlet meniscus volume is used for inlet 3, which results in higher $\Delta P_{\mathrm{B} 2}$ and $\Delta V_{\mathrm{B} 2}$.

\subsection{The effect of increasing step on reducing the backflow}

So far, we have studied the backflow in surface tension-driven networks with three inlets and three channels. Now, we expand our understanding to surface tension-driven networks with five inlets and five channels with one junction. Fig. 5a shows the circuit diagram of the surface tension-driven network. Solution $i$ was injected at inlet $i$ in step $i$, and the

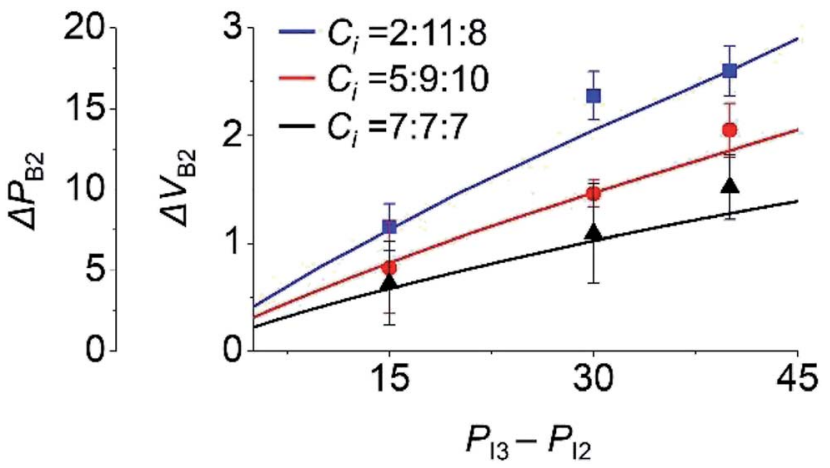

Fig. 4 Effect of initial pressure $\left(P_{\mathrm{li}}\right)$ of inlet $i$ in step 3 on the backflow of inlet 2. Lines and points are the theoretical and experimental $(n=3)$ values, respectively.

initial pressures of inlet $i$ in step $i$ were 35, 35, 75, 75, and $75 \mathrm{~Pa}$, respectively. The left to right panels of Fig. $5 \mathrm{~b}$ show the change in $\Delta P_{\mathrm{B} i}$ and $\Delta V_{\mathrm{B} i}$ at inlet $i(i=1$ to 3$)$. For example, inlet 4 (right panel) shows the changes in $\Delta P_{\mathrm{B} 4}$ and $\Delta V_{\mathrm{B} 4}$ only in step 5 without steps 3 and 4 . This is because the injection to inlet 4 occurs in step 4 , and inlet 4 is empty before step 4 . Similarly,

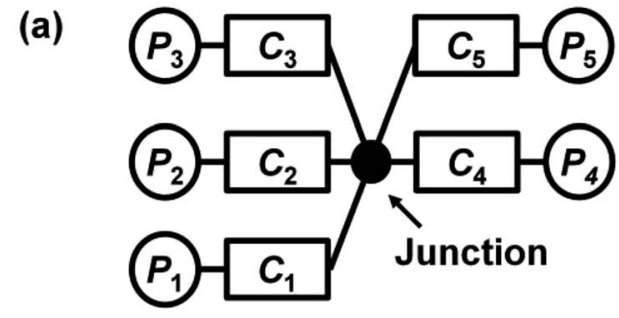

(b)

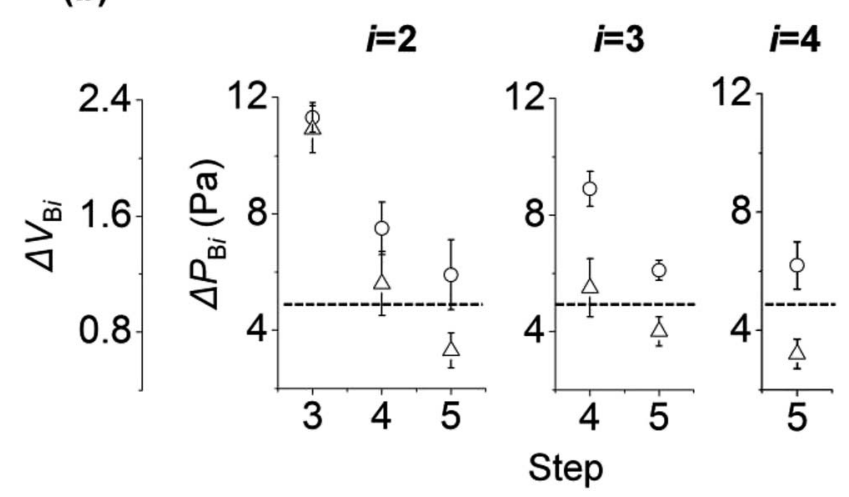

Fig. 5 Backflow in surface tension-driven networks with five inlets and five channels. (a) Circuit diagram of the surface tension-driven network. (b) Change of $\Delta P_{\mathrm{B} i}$ and $\Delta V_{\mathrm{B} i}$ at inlet $i$ ( $i=2$ to 4). Symbols designate subsets: circles for case 1 ; triangles for case 2 . In case $1, C_{j}=$ $5,10,9,13$, and $13\left(\times 10^{-12}\right) \mathrm{m}^{5} \mathrm{~N}^{-1} \mathrm{~s}^{-1}(j=1$ to 5$)$. In case $2, C_{j}=5,10$, $9,5,4\left(\times 10^{-12}\right) \mathrm{m}^{5} \mathrm{~N}^{-1} \mathrm{~s}^{-1}$. For both cases, $r_{j}=2,1,1,1$, and $1 \mathrm{~mm}$. For $\Delta V_{\mathrm{B} i}<1$ (under the dashed line), the backflow of inlet $i$ stays in channel $i$ and does not go into other channels. The error bars were obtained from five experiments. 
inlet 3 (middle panel) shows the variations in $\Delta P_{\mathrm{B} 3}$ and $\Delta V_{\mathrm{B} 3}$ only in steps 4 and 5, without step 3 .

For inlets 2 and $3, \Delta P_{\mathrm{B} i}$ and $\Delta V_{\mathrm{B} i}$ decrease as the step number increases. The initial pressures of inlets increase with increasing step number. This is because by adding solutions to inlets, the volumes of inlet menisci increase as the step number increases. On the other hand, we set the initial pressure of inlet $i$ in step $i$ ( $i=3$ to 5 ) as constant at $75 \mathrm{~Pa}$. As a result, the initial pressure difference between inlet $i$ and the other inlets in step $i$ decreased. In Fig. 4, we explained that the backflow pressure at inlet 2 decreases if the pressure difference between $P_{\mathrm{I} 3}$ and $P_{\mathrm{I} 2}$ is reduced in step 3. Thus, similar to the case in Fig. $4, \Delta P_{\mathrm{B} i}$ and $\Delta V_{\mathrm{B} i}$ decrease with increasing step number. In addition, if $\Delta V_{\mathrm{B} i}$ is under the dashed lines $\left(\Delta V_{\mathrm{B} i}<1\right)$ in Fig. $5 \mathrm{~b}$, then the backflow of inlet $i$ stays in channel $i$ and does not go into other channels.

In addition, our model is broadly applicable to analyze the backflow of the paper microfluidic devices as well as polymer devices. This is because, even for a paper device with porous layer, its inlet pressure can be modeled as variable pressure source by Laplace pressure and its channels can be modeled as fluidic conductor. Specifically, although paper channels follow Darcy's law and polymer channels obey Poiseuille's law, in the two laws flow rate $(Q)$ is commonly proportional to pressure difference $(\Delta P)$. That is, $Q=C \Delta P$, where $C$ is fluidic conductance of a channel. $C$ is $A k /(\mu L)$ in a paper channel and is $\pi r^{4} /$ $(8 \mu L)$ in a polymer channel with circular cross section. Here, $A$ is cross sectional area of channel, $\mu$ is viscosity, $L$ is filled channel length, $k$ is permeability, $r$ is channel radius. Thus, because of the similarity between the two channel types, our model is applicable to paper microfluidic devices.

\section{Conclusion}

Our main contributions for a surface tension-driven network, where the pressure difference of the inlet menisci drives fluidic motions, are as follows: (i) developing a detailed model that describes the backflow strength of the surface tension-driven network; (ii) analyzing the contributions of network parameters (e.g., fluidic conductance of channels, inlet radius, and initial pressure of inlets) to the backflow strength; (iii) characterizing the nonlinear relation between backflow volume and backflow pressure by the change of inlet radius; and (iv) presenting the change in backflow pressure with increasing step numbers.

We showed that each network element, including channel and inlet, has distinct characteristics that affect the change in inlet pressure. A large inlet size makes the change in the corresponding inlet pressure slow. Low fluidic conductance of a channel also makes the pressure change of the inlet directly connected to the channel slow. These two characteristics result in the complex behaviors of inlet pressures when the network elements constitute a surface tension-driven network. Backflow pressure decreases with the decrease (increase) in the corresponding fluidic channel conductance (inlet size). On the other hand, backflow volume reaches its peak value in the middle of the radius change of the corresponding inlet. This is because the inlet pressure was strongly affected by the radius change, but other inlet pressures were insignificantly influenced. In addition, we showed that backflow strength decreases when the initial pressure difference between the inlets decreases. Finally, we showed that in networks consisting of five inlet-channel elements, backflow pressure decreases as the step number increases because the pressure difference between the inlet where the solution is injected and the other inlets decreases. Findings in this study provide useful guidelines for controlling backflow in surface tension driven networks. Further studies could be performed to analyze and prevent backflows in more complex microfluidic networks with multiple junctions.

\section{Conflicts of interest}

There are no conflicts of interest to declare.

\section{Acknowledgements}

This study was supported by Konkuk University in 2016.

\section{References}

1 G. M. Walker and D. J. Beebe, A passive pumping method for microfluidic devices, Lab Chip, 2002, 2(3), 131-134.

2 E. Berthier and D. J. Beebe, Flow rate analysis of a surface tension driven passive micropump, Lab Chip, 2007, 7(11), 1475-1478.

3 A. Javadi, M. Habibi, F. S. Taheri, S. Moulinet and D. Bonn, Effect of wetting on capillary pumping in microchannels, Sci. Rep., 2013, 3, 1412.

4 T. T. Al-Housseiny, P. A. Tsai and H. A. Stone, Control of interfacial instabilities using flow geometry, Nat. Phys., 2012, 8(10), 747.

5 E. Elizalde, R. Urteaga and C. L. Berli, Rational design of capillary-driven flows for paper-based microfluidics, $L a b$ Chip, 2015, 15(10), 2173-2180.

6 E. Elizalde, R. Urteaga, R. R. Koropecki and C. L. Berli, Inverse problem of capillary filling, Phys. Rev. Lett., 2014, 112(13), 134502.

7 M. Zimmermann, H. Schmid, P. Hunziker and E. Delamarche, Capillary pumps for autonomous capillary systems, Lab Chip, 2007, 7(1), 119-125.

8 V. G. Levich and V. S. Krylov, Surface-tension-driven phenomena, Annu. Rev. Fluid Mech., 1969, 1(1), 293-316.

9 E. Boucher, Capillary phenomena: properties of systems with fluid/fluid interfaces, Rep. Prog. Phys., 1980, 43(4), 497.

10 J. W. Van Honschoten, N. Brunets and N. R. Tas, Capillarity at the nanoscale, Chem. Soc. Rev., 2010, 39(3), 1096-1114.

11 I. Meyvantsson, J. W. Warrick, S. Hayes, A. Skoien and D. J. Beebe, Automated cell culture in high density tubeless microfluidic device arrays, Lab Chip, 2008, 8(5), 717-724.

12 J. Warrick, I. Meyvantsson, J. Ju and D. J. Beebe, Highthroughput microfluidics: improved sample treatment and washing over standard wells, Lab Chip, 2007, 7(3), 316-321. 
13 T. J. Clark, P. H. McPherson and K. F. Buechler, The triage cardiac panel: Cardiac markers for the triage system, Point of Care, 2002, 1(1), 42-46.

14 E. Delamarche, D. Juncker and H. Schmid, Microfluidics for processing surfaces and miniaturizing biological assays, Adv. Mater., 2005, 17(24), 2911-2933.

15 M. Choi, Y. Na and S. J. Kim, Hydrophilic strips for preventing air bubble formation in a microfluidic chamber, Electrophoresis, 2015, 36(23), 2896-2901.

16 A. Olanrewaju, M. Beaugrand, M. Yafia and D. Juncker, Capillary microfluidics in microchannels: from microfluidic networks to capillaric circuits, Lab Chip, 2018, 18(16), 2323-2347.

17 S.-J. Kim, S. Paczesny, S. Takayama and K. Kurabayashi, Preprogrammed, parallel on-chip immunoassay using system-level capillarity control, Anal. Chem., 2013, 85(14), 6902-6907.

18 S.-J. Kim, S. Paczesny, S. Takayama and K. Kurabayashi, Preprogrammed capillarity to passively control system-level sequential and parallel microfluidic flows, Lab Chip, 2013, 13(11), 2091-2098.

19 S.-J. Kim, Y. T. Lim, H. Yang, Y. B. Shin, K. Kim, D.-S. Lee, S. H. Park and Y. T. Kim, Passive microfluidic control of two merging streams by capillarity and relative flow resistance, Anal. Chem., 2005, 77(19), 6494-6499.

20 A. R. Rezk, A. Qi, J. R. Friend, W. H. Li and L. Y. Yeo, Uniform mixing in paper-based microfluidic systems using surface acoustic waves, Lab Chip, 2012, 12(4), 773-779.

21 Y. Zhai, A. Wang, D. Koh, P. Schneider and K. W. Oh, A robust, portable and backflow-free micromixing device based on both capillary-and vacuum-driven flows, $L a b$ Chip, 2018, 18(2), 276-284.

22 E. Berthier, J. Warrick, B. Casavant and D. J. Beebe, Pipettefriendly laminar flow patterning for cell-based assays, $L a b$ Chip, 2011, 11(12), 2060-2065.

23 Y. Lee, M. Choi and S.-J. Kim, Method to prevent backflow in a capillarity network for bioassays: Exploiting time constant ratios, Sens. Actuators, B, 2018, 255, 3630-3635.

24 A. Gliere and C. Delattre, Modeling and fabrication of capillary stop valves for planar microfluidic systems, Sens. Actuators, A, 2006, 130, 601-608.

25 Y. Xia and G. M. Whitesides, Soft lithography, Angew. Chem., Int. Ed., 1998, 37(5), 550-575.

26 P. J. Resto, E. Berthier, D. J. Beebe and J. C. Williams, An inertia enhanced passive pumping mechanism for fluid flow in microfluidic devices, Lab Chip, 2012, 12(12), 22212228.

27 J. Ju, J. Y. Park, K. C. Kim, H. Kim, E. Berthier, D. J. Beebe and S.-H. Lee, Backward flow in a surface tension driven micropump, J. Micromech. Microeng., 2008, 18(8), 087002.

28 P. J. Resto, B. J. Mogen, E. Berthier and J. C. Williams, An automated microdroplet passive pumping platform for high-speed and packeted microfluidic flow applications, Lab Chip, 2010, 10(1), 23-26.

29 B. Lavi, A. Marmur and J. Bachmann, Porous media characterization by the two-liquid method: effect of dynamic contact angle and inertia, Langmuir, 2008, 24(5), 1918-1923. 